Session 2630

\title{
Physics in the Integrated First Year; Second Attempt
}

\author{
William H. Bassichis \\ Department of Physics \\ Texas A\&M University \\ College Station, TX 77843
}

\section{Introduction}

Texas A\&M University is part of a seven school program, supported by the National Science Foundation, called the Foundation Coalition, FC. One of the goals of the FC is to integrate the freshman year courses taken by engineering undergraduates. The First Year Integrated Program at Texas A\&M University was implemented in 1994 and initially combined the study of engineering, graphics, calculus, physics, and English. Later, the first course in chemistry was added to the program. The first semester then consisted of 2 credits in engineering, 2 in graphics, 4 in mathematics, 2 in physics and 2 in English. The second semester consisted of 1 credit in engineering, 1 in graphics, 4 in mathematics, 2 in physics, 1 in English and 4 in chemistry. Two sections of about 50 students each were chosen somewhat randomly from those entering freshman who were judged to be calculus ready. After completing the Freshman year the performance of these students was compared with that of students enrolled in the traditional program and in all aspects they either equaled or surpassed the performance of traditional students. Since the FC students had also greatly enhanced their teaming skills, another goal of the program, it was decided to continue the program the second year and to double the number of first year students participating.

\section{The Need for Modification}

There were a number of changes that had to be made in the program if it were to continue and if the size were to be increased to 200 students. It was deemed uneconomical to maintain a class size less than one half that in the traditional program, therefore the classes would each need to have at least 100 students. Thus the room which had been rather laboriously designed for optimal teaming and use of technology, and which had been considered a rather vital element of the program, was abandoned. A traditional lecture hall was slightly modified so that teaming was at least possible, if inconvenient. The content of the physics course had to be modified because in the design of the second year curriculum it was assumed that students had been exposed to the concepts of charge and current and could solve simple circuit problems. As this material was needed in the beginning of the first semester of the second year and is not covered in the beginning of the usual course in E\&M, it had to be included in the first year. However, requiring that first year students take two semesters of physics, the traditional mechanics followed by the usual E\&M, is simply not consistent with an integrated curriculum that begins with beginning calculus. This presented a major problem.

The fact that in the first attempt at an integrated curriculum there was only one half of a physics course per semester had other deleterious effects. The material was often too diluted in time which led to difficulties in comprehension. Furthermore students often ignored physics in order to concentrate their efforts on those subjects that counted more. This was an entirely reasonable behavior having disastrous consequences since other courses relied on the students' mastery of the material covered in physics.

The final reason for modifying the rather successful physics component was financial. If, as part of a teaching load, a faculty member were assigned one traditional physics course for engineering students, the number of students involved is approximately 100 per semester. If instead that person taught in the integrated program, even at the level of 100 students per class, it would take two semesters of teaching the half courses to obtain the same number of credit hours. Thus, to service the same number of students would require either twice the 
number of faculty or the same number teaching twice as many classes. Since the integrated curriculum is more demanding of time than a traditional course, independent of the number of contact hours, the latter alternative would mean the end of the program. Unfortunately using twice the number of faculty for the same number of students was not considered economically feasible.

The challenge was thus to modify the physics component of the successful first year program so that the integration would be maintained, twice the credit, and thus twice the number of hours, in physics be included, and the fundamentals of E\&M be covered in addition to that material covered previously.

\section{The Content of the Revised Physics Component}

Traditional engineering students have a semester course in mechanics, with possibly some heat and sound, followed by a semester course in E\&M, possibly with some optics. As is known from the theory of education if you don' t use it, you lose it and the time separation between the introduction of concepts in mechanics and their possible application in E\&M is long enough to render these concepts lost. Therefore when teaching electricity and magnetism instructors generally do not rely on the student's knowledge of mechanics, to any great extent. In fact much of E\&M is just an application or extension of mechanics and the two subjects can be integrated in a way that would enhance the study of both. There are other advantages to this integration. The student will be prepared for their second year courses and, with physics behind them, will have more time for other courses. The new material can integrated into the present, integrated physics, math, engineering, chemistry, and English without a large perturbation, and it would enhance and enrich the program. Furthermore it solves problems connected to funding of faculty and coverage of material which have no other apparent solution.

In the first attempt at integration of the curriculum, the first three weeks of physics were given to engineering, to be paid back at a later time. This enabled the mathematics instructors to at least introduce differentiation and integration before these mathematical concepts were employed in kinematics. In the scaled up version of the program this flexible scheduling, which was used to considerable advantage, became a scheduling nightmare. Each course had to meet on a regular basis in order to accommodate room assignments, particularly for laboratories, and availability of graduate teaching assistants, as well as faculty. In this second attempt the first week was devoted to teaming training, as in the past. In the second and third weeks Geometrical Optics was studied, both theoretically and in the laboratory. This material was easily mastered, was useful in teaching problem solving techniques, introduced the students to the essentials of laboratory work, refreshed their trigonometric memories, and demonstrated that the laws of nature as expressed abstractly could be put to experimental test and be verified. The first integrated exam, an essential ingredient of the FC program at Texas A\&M, was concerned with the optimal orientation of a reflecting part of a solar oven, and this convinced the students that the concepts studied in physics were of practical importance in their engineering education. The study of kinematics, utilizing the concepts introduced in mathematics, was then began, as in the first integrated program. Again the equations derived in physics were rather immediately applied by the engineering faculty to 'realistic' problems so that the connection between mathematics, physics and engineering was reinforced. After the discussion of Newton's Laws, various forces are traditionally considered, namely the force exerted by a spring, friction, gravity, etc. This was deemed the appropriate time to bring in the observations connected to charges and Coulomb's Law. In fact, because it is the same functional form as Newton's Law of Gravitation, the problems connected to the two force laws are almost identical, just changing charge for mass. Thus both forces were used in problems designed to learn about forces, vectors, and Newton's Law and the student rather painlessly gained an understanding of Coulomb's Law. This was followed by the usual detailed study of Newton's Laws. The notion of the gravitational field is usually not given much, if any, attention in mechanics because it does not play an essential role in the development of the laws of physics. Electric fields, on the other hand, play an essential role in the development of the laws of E\&M. In fact there is a benefit to first introducing fields associated with mass, since this is a more familiar concept, and following that by the introduction of electric fields in exactly the same manner. This makes the idea of an electric, vector field easier to understand. The notion of superposition, whether of gravitational or electric forces, is now introduced with systems of discrete, point objects followed by consideration of continuous distributions either of charge or mass. These calculations were done analytically in physics class and were used as exercises in mathematics for practice in solving 'summation of effects' problems using Maple. 
At this point the mathematics component has introduced the notion of a path integral. This sets the stage for a very rigorous and detailed study of work, potential energy functions, the concept of conservative and nonconservative forces, and Conservation of Energy. As this is perhaps the most sophisticated and difficult concept encountered in the entire first year, a large amount of time is spent on this material. Since voltage is just electric potential energy per unit charge, this is the natural place to introduce this idea and the work done by various electric fields were used as examples of the general equations. Because both the force of gravity and Coulomb's force are simplest in polar coordinates, these are introduced in mathematics and then used in physics to calculate the corresponding electric potential functions which are then employed in various applications, such as conservation of energy problems with charged particles in electric fields. One does not generally calculate gravitational potential energy for continuous distributions of mass as one does for charges, but there is no reason why not, as it would help the student understand electric potentials and would provide additional applications of the process of integration studied in calculus.

The first semester ends with a rigorous derivation of Gauss' Law for particles interacting either with Coulomb's force or the gravitational force. One does not normally consider mass distributions in this way in mechanics but this again aids in the understanding of the application of Gauss' Law to charge distributions and in itself has some interesting applications. The usual spherical, cylindrical and planar symmetric situations are considered. This follows immediately after the study of surface integrals in calculus thus providing motivation for and practice with the operation.

The second semester begins with the study of the first real electrical device, namely the capacitor. This provides the opportunity to utilize the notions of work and electric potential difference as a line integral and, for the cases of spherical and cylindrical capacitors, utilizes the knowledge of polar coordinates previously attained. At this point simple experiments with circuits containing capacitors and batteries are used to connect the abstract concepts with reality. The emphasis is shifted back to mechanics with the study of systems of particles leading to the law of Conservation of Momentum. The focus returns to E\&M with the introduction of the electrical concepts of current, resistance, Ohm's Law and the study of simple circuits. This study is reinforced by the utilization of extremely simple experiments involving batteries and resistors. The connection between the 'voltage loop equation' and the path integral of the electric field can be demonstrated which again reinforces the knowledge of work gained in mechanics.

Magnetic fields and the forces they exert on charged particles are studied at this point. Magnet devices such as spectrometers, with the circular motion of the charged particles, are used to motivate the return to the study of kinematics in polar coordinates. The radial and tangential components of the velocity and acceleration vectors are derived in great detail as this can then be used in the study of angular momentum. Torque is introduced with the torque on a current carrying loop, such as in a generator or motor, as an example. The law of Conservation of Angular Momentum can now be obtained, again by considering a system of particles. The consideration of extended objects is carried out in conjunction with mathematics where moments of inertia are calculated in various ways as yet another application of integration.

Faraday's Law is now introduced and is contrasted with the conservative forces and fields previously encountered. This example of a non-conservative force will serve to review and reinforce the study of conservative and non-conservative forces and the existence of potential energy functions. This is presently the most difficult part of mechanics, conceptually, and this example can be very helpful.

At this point the integration of mechanics and E\&M takes its most natural form. The mass on a spring and other oscillating systems can be studied both theoretically and experimentally and Faraday's Law can be applied to simple circuits which can also be studied in the lab. The resulting equations for the mechanical and electromagnetic systems, which are mathematically identical, will be treated in detail in the mathematics component both analytically and using the computer. Since there are engineering applications that will be introduced at this time this should be integration at its most intense.

The remainder of the second semester is devoted to the development of laws of thermodynamics, as in the present first year program. A kinetic theory approach is used in order to utilize the connection between mechanics and thermodynamics. By integrating with the chemistry component those ideas normally treated in 
both physics and chemistry such as temperature scales, ideal gas laws, etc. can be utilized immediately in the introduction to thermodynamics and thus be reinforced rather than repeated. The appearance of the same mathematics, such as the path integral, in the three areas of physics will facilitate the student's understanding of the subtle concepts involved. Even the study of entropy will look less foreign because of the similarity with definition of the potential energy function in mechanics and the electric potential function in E\&M and the use of entropy in chemistry in the discussion of the phases of matter.

\section{The Results at this Point}

At the time this paper is being prepared the first semester has been completed except for the final exams. The performance on these exams compared to that of traditional students will provide interesting preliminary results but, because of the difference in the sequence in which topics are covered, a comparison of exam results after the second semester will be more meaningful. At this time, however, a number of conclusions can be drawn. The level of enthusiasm of these students far surpasses that of students in a traditional physics course for engineering students. The fact that there are 100 students in each class, rather than 50 as was the case last year, does not seem to have adverse effects on the students, although the difficulty in learning students' names seems to rise at least with the square of the number in the class. The ability of the students to work in teams of four does not seem to be effected by the poor geometric layout in the room this year, compared to the optimal table shapes and size utilized the first year. Most important, the performance of these students on exams, though never as high as one would hope, is significantly higher than that being demonstrated in the traditional physics courses being taught to engineering students.

Finally there was some fear that the students would not be ready to comprehend the E\&M concepts normally deferred until the second semester of physics. Indeed the students did experience difficulty with some of this material but it was not any greater than is encountered in the traditional course with the concepts of torque and angular momentum which are taught in the first semester. These topics will be treated in the second semester in this modified approach. 